\title{
Intentions: A Confident-Based Interaction Design for Smart Spaces
}

\author{
Mario Vega-Barbas and Miguel A. Valero \\ T>SIC group, Universidad Politécnica de Madrid, Spain \\ \{mvega, mavalero\} @diatel.upm.es
}

\begin{abstract}
The paradigm of ubiquitous computing has become a reference for the design of Smart Spaces. Current trends in Ambient Intelligence are increasingly related to the scope of Internet of Things. This paradigm has the potential to support cost-effective solutions in the fields of telecare, e-health and Ambient Assisted Living. Nevertheless, ubiquitous computing does not provide end users with a role for proactive interactions with the environment. Thus, the deployment of smart health care services at a private space like the home is still unsolved. This $\mathrm{PhD}$ dissertation aims to define a person-environment interaction model to foster acceptability and users confidence in private spaces by applying the concept of user-centred security and the human performance model of seven stages of action.
\end{abstract}

\section{$1 \quad$ Research Situation}

The presented work is framed into the context of TALISEC+ project $^{1}$ and supported up to 2015 by a PhD scholarship (FPI) granted by the Spanish Ministry of Economy and Competitiveness. Currently I have completed my training period (MSc) that led to the beginning of the research stage of my thesis. Also this thesis is going to be support by a double doctorate cooperation agreement between the Royal Institute of Technology (KTH) from Sweden and Universidad Politécnica de Madrid from Spain.

Along the training period of my PhD studies I was able to specify the problem domain of my research as well as the expected results of my thesis work. Besides, a brief stay in the Smart Environment Research Group at the University of Ulster allowed me the validation of the objectives detailed below. However, expert researchers about Human-Computer Interaction (HCI) can contribute in an effective way to enhance the validity and feasibility of this work. This is my motivation to attend to the Doctoral Consortium of SouthCHI 2013.

\footnotetext{
${ }^{1}$ http: //www.morelab.deusto.es/talisman/index_en.html
} 


\section{Background and Motivation}

Current HCI models aim to look at the person as the center of all interaction activities [1]. Ubiquitous computing (ubicomp), smart spaces and the applications and services envisaged for these domains need to define new users' roles [2]. It is complex to include human beings in the traditional computational loop of these scenarios without degrading their capabilities [2][3].

Some studies analyze the implications of the inclusion of ubicomp into living spaces such as the home [4]. The majority of papers do focus on improving other required aspects like interoperability, devices self-discovery or the capability to program smart spaces in the Future Internet [5][6]. Thus, the field of ubicomp interaction has been dealt in an isolated way from a viewpoint of services and applications which does not include the end user [3][7]. Therefore, a new global approach needs to be defined in order to situate the user in the requested place.

Usage of ubicomp and proactivity in daily routine at health care area is still a challenge [4][7]. Telecare and e-health services could be enhanced by ubicomp if and only if users accept this emerging interaction way.

The aim of this PhD work is the creation of a new Person-Smart Space interaction model that maximizes users' acceptance and confidentiality of pervasive healthcare and e-health services. This research addresses to identify those factors that should guide a new model and its application in the paradigm of ubiquitous computing, enhancing other acceptance model such as Pervasive Technology Acceptance Model (PTAM) [8] and Technology Acceptance Model 3 (TAM) [9].

\section{$3 \quad$ Statement of Thesis}

This thesis tries to solve the intrinsic problem that arises from the inclusion of ubiquitous and proactive technology in homes or private environments where personal information is managed. Consequently, the following problems are stated:

1. Identification of indicators that specify the level of confidence and acceptance of a person in relation with the application of pervasive technology at home.

2. Specification of suitable interaction models to support users at ubiquitous computing home based scenarios that manage confidential data such us e-health, telecare and Ambient Assisted Living.

3. The definition and implementation of hardware and software modules requested by a smart home e-health or telecare service to provide the expected confidence.

4. Finally, verification and validation of the interaction model developed. This process will be done using three methods of evaluation tools: interviews and group dynamics, validation through use cases and scenarios and prototyping into real deployments at several research projects. 


\section{$4 \quad$ Research Goals and Methods}

The presented $\mathrm{PhD}$ dissertation would pursues the following goals:

- Goal 1. To define a measurable set of indicators capable of describing the willingness and barriers of a person, related to confidence, in order to accept a ubiquitous computing service at a smart home environment. The level of achievement of this goal will be validated in telecare and e-health domains.

Previous experiences will be reviewed to extract an initial set of indicators about users' attitudes towards confidentiality and privacy in ubicomp scenarios. Then, guided interviews with end users of telecare and e-health services will be carried out in order to fine-tune these indicators and validate its application.

- Goal 2. To set out a scalable interaction model in private ubicomp scenarios that integrates users' roles and attitudes. This model should enhance the acceptability and confidence of users by providing an accessible way to understand and verify the behavior of his surrounding environment.

Published results from related field works as well as the set of indicators defined in Goal 1 will be the basis to design the interaction model. Next, a validation home telecare or e-health service will be deployed including the requested interaction facilities, software and hardware, that provides end users with expected confidence. In this way, its verification and validation will be done through a proof of concept to be deployed and evaluated with end users into real scenarios such as their own homes and the Accessible Digital Home sited at Technical University of Madrid ${ }^{2}$. Moreover, the model developed will be use in order to supply prototypes for several e-health research projects at KTH, Sweden. Those prototypes and group dynamics and interviews help me to refine and validate the proposal.

The presented work should furnish guides or interaction models to guarantee an optimal level of user understanding of what happens in a smart environment. The result should contribute with a new approach to defining user roles in ubiquitous computing and should also lead to new ways of designing and developing smart environments designed for the people.

\section{$5 \quad$ Dissertation Status}

To date there have been two interviews to a set of users to determine which technologies should be used to make the process of interaction within homes and a survey about the confidence on smart spaces. Additionally, we have finished a complete study about confidence into smart environments with 200 people. With this information I have could define and enhance a user model for the specific case of telecare in the home with four different types of players (inhabitants, technology

\footnotetext{
${ }^{2}$ http: / / hogardigitalaccesible.euitt.upm.es/
} 
integrators, services providers and other caregivers as family). Furthermore, I have designed software and hardware architecture to support this future interaction model based on actions and intentions, following the schema shown in the Fig. 1.

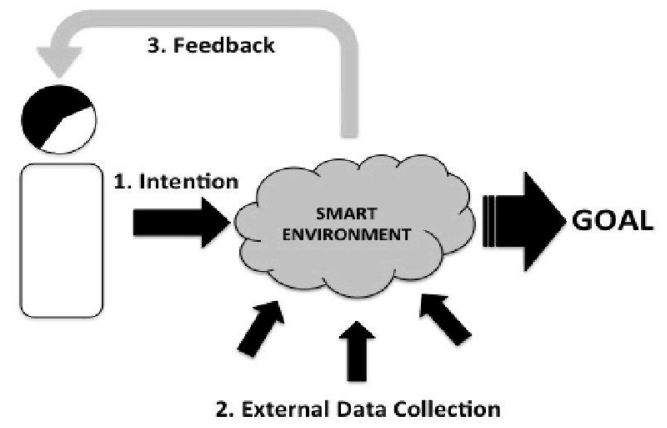

Fig. 1. Intention-based Interaction Model

This base work has been contrasted by a number of congress contributions. Additionally, through a research brief stay in the SERG group at the University of Ulster, has been able to verify the feasibility and validity of the aforementioned architecture design. Besides, the Suggestive Autonomy ${ }^{3}$ research project that the author was co-director put into practice the basic ideas of this doctorate.

\section{References}

1. Sharp, H., Rogers, Y., Preece, J.: Interaction Design: Beyond Human-Computer Interaction, 3rd edn. Wiley Publishing (2012)

2. Tennenhouse, D.: Proactive computing. Commun. ACM 43(5), 43-50 (2000)

3. Poslad, S.: Ubiquitous Computing: Smart Devices, Environments and Interactions. Wiley Publishing (2009)

4. Bohn, J., Coroama, V., Langheinrich, M., Mattern, F., Rohs, M.: Social, Economic, and Ethical Implications of Ambient Intelligence and Ubiquitous Computing. Journal of Human and Ecological Risk Assessment 10(5), 763-786 (2004)

5. King, J., Bose, R., Yang, H., Pickles, S., Helal, A.: Atlas - A Service-Oriented Sensor Platform. In: Proceedings of the First IEEE International Workshop on Practical Issues in Building Sensor Network Applications (SenseApp 2006) (2006)

6. Kuniavsky, M.: Smart Things: Ubiquitous Computing User Experience Design. MK Publishers (2010)

7. Aarts, E., Ruyter, B.: New Research Perspectives on Ambient Intelligence. Journal of Ambient Intelligence and Smart Environments 1(1), 5-14 (2009)

8. Connelly, K.: On Developing a Technology Acceptance Modelfor Pervasive Computing. In: Proceedings of Ubiquitous System Evaluation (USE) at the Ninth International Conference on Ubiquitous Computing (UBICOMP) (2007)

9. Venkatesh, V., Bala, H.: TAM 3: Advancing the Technology Acceptance Model with a Focus on Interventions. Manuscript in-preparation

\footnotetext{
${ }^{3}$ http://www . autonomiasugestiva.es
} 